\title{
The wavelength reconstruction from toroidal spectrometer image data
}

\author{
J. Blazej ${ }^{1}$, M. Tamas ${ }^{1}$, L. Pina ${ }^{1}$, A. Jancarek ${ }^{1}$, S. Palinek ${ }^{1}$, P. Vrba ${ }^{2}$ \\ \& M. Vrbova ${ }^{1}$ \\ ${ }^{I}$ Czech Technical University in Prague, Czech Republic \\ ${ }^{2}$ Institute of Plasma Physics, Academy of Science, Czech Republic
}

\begin{abstract}
We are reporting spectroscopy studies of Nitrogen filled capillary discharge. The identification procedure of spectra was carried out with the use of an extreme ultraviolet grazing incidence spectrometer with toroidal grating. The source of radiation and the object under study is a pinching alumina capillary discharge in Nitrogen, where stimulated emission in the $13.4 \mathrm{~nm}$ wavelength region is expected. To simplify the alignment of the spectrometer during the experiment the off Rowland circle registration scheme is used. In this scheme, spectra are recorded in a single plane and thus exact focusing of the input slit takes place only for one single wavelength, which corresponds to the intersection of the plane of registration with the Rowland circle. Appropriate image processing must be applied to reconstruct the spectra profile and to calibrate wavelength positions. The cooled extreme ultraviolet sensitive CCD camera was used in the role of planar detector. It has a $512 \times 512$ elements matrix with $24 \mu \mathrm{m}$ square pixels. The 16-bit dynamic range together with very low-noise provide a good data source for image post-processing. The experimental impossibility to record simultaneously zeroth order maximum and the first diffraction order spectrum was a crucial problem in the presented process. The presented methodology can be applied to any experiment using toroidal grating spectrometer.

Keywords: spectrometer, image distortion, calibration.
\end{abstract}

\section{Introduction}

We are reporting spectroscopy studies of Nitrogen filled capillary discharge. The goal of our work is to create a technique for routine use of a spectrometer with 
toroidal grating with relatively small image detector in the off Rowland circle registration scheme. The usual type of detector for this scheme is a linear CCD that allowing images simultaneously zeroth and first order maximum and that in principle eliminate geometrical distortion of lines aside the circle plane. Spectra acquired by a planar CCD are distorted by non-ideal imaging in the spectrograph and non-linear dispersion must be eliminated to reconstruct wavelength information.

The first problem is that geometrical distortion of acquired spectra origins from imaging of input slit to a plane. In standard spectrograph configuration the output slit is used to overcome this problem. This is an on Rowland circle registration scheme. The other possibility is to use a line (linear) CCD without output slit in the plane of the Rowland circle. In this case the distortion is converted into modification of dispersion. To improve the signal to noise ratio and overcome experimental difficulties with low detection yield and energy budget we decided to use a planar CCD.

\section{Distortion elimination}

The spatial configuration of the spectrometer with toroidal grating is schematically drawn in figure 1. For the theoretical review see Haber [1]. The cross section of the screen with the Rowland circle defines on preferred wavelength. Its line is imaged without any astigmatism. All others line are distorted in correlation with the distance between the screen and the Rowland circle.
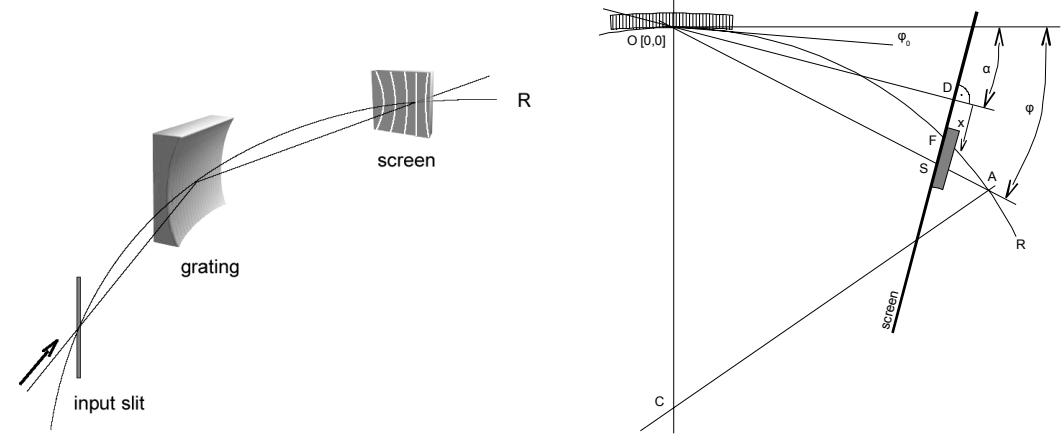

Figure 1: The spatial configuration of the spectrometer with toroidal grating. The input slit is imaging to the screen. The cross section of the screen with the Rowland circle defines on preferred wavelength. It is only one line imaged without any astigmatism. $\varphi$ is the angle of virtual scanning line $p$, the coordinate origin is point $O$. $R$ is the Rowland circle with diameter $R$, center $C$. The angle $\varphi_{0}$ is the zeroth order angle, $\alpha$ is the screen angle. The origin of coordinates at the screen is the point $\mathrm{F}$, the cross-section with the Rowland circle. The distance between grid and screen is called $\mathrm{D}=\mathrm{D}-\mathrm{O}$. 
The analyzed radiation is coming from the left and is mirrored by the grating under grazing angle $\varphi_{0}$. The zero order maximum angle $\varphi_{0}$ is $4^{\circ}$ and the angle of screen is $10^{\circ}$. The coordinate origin is the point $\mathrm{O}$ - the grid center. $\mathrm{X}$ axis is the grid tangent and $\mathrm{y}$ axis is going through point $\mathrm{C}-$ the center of the Rowland circle The screen is at distance $\mathrm{D}$ from the origin, containing the $\alpha$ angle from the $\mathrm{x}$-axis.

To eliminate geometrical distortion of spectra the following method is used. Several horizontal profiles (raw cuts) are taken on the image with good contrast line pattern. From the line pattern several well-defined lines are selected. Each horizontal profile is separately fitted by multiple Gaussian peaks at selected lines. Positions of their centers define a matrix of distortion. Each column of this matrix is fitted by a parabola. The radii of curvature of these parabolas are changing for different columns and this dependence has a minimum. The position of this minimum can be used for absolute calibration. The generated matrix can be used for distortion elimination after its smoothing by the parabola fit. For distortion elimination - image warping - we are using open source program xmorph [2] with sophisticated wavelet amplitude correction to ensure the photometric data from original spectra. The result of these steps is an image with parallel spectral lines over the entire screen, see figure 2 .
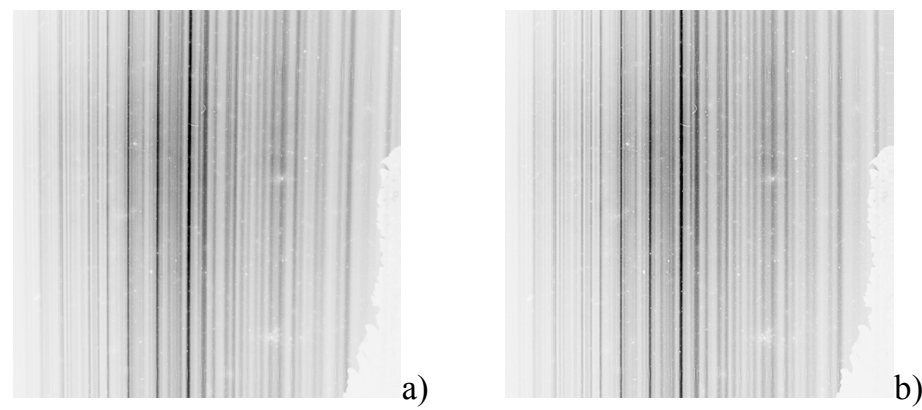

Figure 2: $\quad$ The negative of the primary picture taken by CCD (a) and final warped image (b). All its lines are straight and parallel. Optionally the flat field and background correction can be applied to enhance the signal to noise ratio and recognize and eliminate the dead pixels.

\section{Calibration}

The spectrometer dispersion curve is well known, but the problems of calibration of exposed spectra are two:

1) in real experimental conditions it is impossible to determine the distance $\mathrm{D}$

2) the origin of $x$ axis on screen is unknown, the screen is too small to include zeroth order maximum 
Therefore the experiment has two degree of freedom from the absolute calibration point of view; the position of zeroth order maximum on detector plate and the distance of detector from grating center. Both are experimentally unattainable for us.

The parameters for calibration can be derived from following sources:

\subsection{Calibration filter}

For these wavelengths the L-edge of aluminium can be used. The problem is the absence of continuous source. Some experiment configuration generating a lot of lines can be used, but the final resolution of edge position identification depends on actual spectra. Nevertheless, only one parameter can be evaluated from one edge and filtered spectra were not included in a particular analyzed spectra set.

\subsection{Width of spectral lines}

The width is proportional to focusing. The imaging of spectra to the Rowland circle can be modelled as an imaging by a lens with variable focus length. Assuming a hypothesis about the quasi-Gaussian character of the input signal, the model of dependence of line width on wavelength can be derived. But we are not succeeding to find any algorithm for a reliable line width calculation from spectra with unknown spectral line intensities.

\subsection{Position of non-distorted spectral line on screen}

This invariant reduces the number of degrees of freedom to one defining a relation between searched parameters. The position of the non-distorted line can be estimated with a precision of 10 pixels.

\subsection{Assignment of known wavelengths to bright lines}

The fourth source is the assignment of known wavelengths to bright lines. It is dependent on several presumptions about observed plasma source or on an independent spectral analysis of similar plasma source.

\subsection{Solution}

We decide to use the fourth method. A spectrum identified in this work is generated by a capillary discharge in nitrogen pre-filled alumina capillary (nitrogen pre-fill pressure 0,49 torr), capillary dimensions were $297 \mathrm{~mm}$ long, $3.0 \mathrm{~mm}$ in diameter and the current form is damped sinus with quarter period $\mathrm{t}_{1}=87.5 \mathrm{~ns}$, damping time $\mathrm{t}_{2}=600 \mathrm{~ns}$ and $\mathrm{I}_{0}=22 \mathrm{kA}$. For comparison we have two independently calibrated spectra, see Vrbova et al. [3]. The first one is a spectrum of capillary discharge in nitrogen pre-filled alumina capillary (nitrogen pre-fill pressure 0.9 torr). Capillary dimensions were $56 \mathrm{~mm}$ length and $3 \mathrm{~mm}$ diameter, the current form is damped sinus with quarter period $t_{1}=150 \mathrm{~ns}$, damping time $t_{2}=600 \mathrm{~ns}$ and $\mathrm{I}_{0}=15.5 \mathrm{kA}$. And the second one is a spectrum of ablative capillary discharge in polyoxymethylene (POM) capillary. Capillary 
dimensions were $56 \mathrm{~mm}$ length and $1.1 \mathrm{~mm}$ initial diameter, the current form is damped sinus with quarter period $t_{1}=65 \mathrm{~ns}$, damping time $\mathrm{t}_{2}=150 \mathrm{~ns}$ and $\mathrm{I}_{0}=12$ $\mathrm{kA}$. We have identified a strong resemblance between three spectra. Considering that the only common element present in every discharge is oxygen, there is a strong indication to assign the most intense lines to oxygen ions. By comparing the database [4] and the simulated spectra of different ions made by Sapphire software [5] with measured values we decided to identify the most intense lines around $13 \mathrm{~nm}, 15 \mathrm{~nm}$ and $17.4 \mathrm{~nm}$ as lines belonging to helium like oxygen. Modifying the spectra accordingly we could further identify minor peaks from their absolute wavelength position. By assigning the most intense lines to oxygen ions a very good accordance of measurement with simulated lines of He-like oxygen (O VI) was found. Further very good accordance can be seen with simulated lines of He-like nitrogen $(\mathrm{N} \mathrm{V})$, especially in experiments with higher nitrogen pre-fill pressure.

\section{Conclusion}

The experimental impossibility to record simultaneously zeroth order maximum and the first diffraction order spectrum was a crucial problem in the presented process. We succeeded in eliminating an image distortion and verified its successful calibration. Spectral lines belonging to excited Nitrogen ions $\mathrm{N}^{4+}$ and $\mathrm{N}^{5+}$ were identified. We may note that we were not able to identify as many lines in the interval above $22 \mathrm{~nm}$ as we did in the interval 7-22 $\mathrm{nm}$. This may be caused by lower resolution as we are far away off the Rowland circle. The presented methodology can be applied to any similar experiment using a toroidal grating spectrometer.

\section{Acknowledgements}

This research has been supported by the research framework MSM6840770022, section 3 of Ministry of Education of Czech Republic and experimental data has been obtained thanks to support of grant 1P04LA235.

\section{References}

[1] Haber, H.: The Torus Grating, In: Journal of Optical Society of America vol. 40, number 3, Optical Society of America, Washington DC, USA, March 1950, pp. 153-165.

[2] Mennucci, A.C.G.: Xmorph, [online] <http://xmorph.sf.net>, 2004, compilation gtk2.2 July 17th 2004.

[3] Vrbova, M., Jancarek, A., Vrba, P., Fojtik, A., Scholzova, L., Havlikova, R., Palinek, S., Soft X-ray Emission from Nitrogen Capillary Discharge, ICXRL Peking 2004.

[4] NIST Atomic Spectra Database Lines Form, [online], $<$ http://physics.nist.gov/PhysRefData/ASD/lines_form.html $>$.

[5] Sapphire professional, version 1.0.3.3 (Cavendish Instruments Ltd. 2003). 